\title{
Turbulent Drag Reduction by Flexible and Rodlike Polymers: Crossover Effects at Small Concentrations
}

\author{
Emily S.C. Ching \\ Dept. of Physics, The Chinese University of Hong Kong, Shatin, Hong Kong \\ T. S. Lo and Itamar Procaccia \\ Dept. of Chemical Physics, The Weizmann Institute of Science, Rehovot 76100, Israel
}

\begin{abstract}
Drag reduction by polymers is bounded between two universal asymptotes, the von-Kármán loglaw of the law and the Maximum Drag Reduction (MDR) asymptote. It is theoretically understood why the MDR asymptote is universal, independent of whether the polymers are flexible or rodlike. The cross-over behavior from the Newtonian von-Kármán log-law to the MDR is however not universal, showing different characteristics for flexible and rodlike polymers. In this paper we provide a theory for this cross-over phenomenology.
\end{abstract}

\section{INTRODUCTION}

The phenomenon known as "drag reduction" by polymers in turbulent channel flows [1, 2] is conveniently discussed in channel geometry for fixed pressure heads, such that the reduction in the drag is manifested as an increased mean velocity. For the sake of comparison between different fluids it is convenient to choose normal-

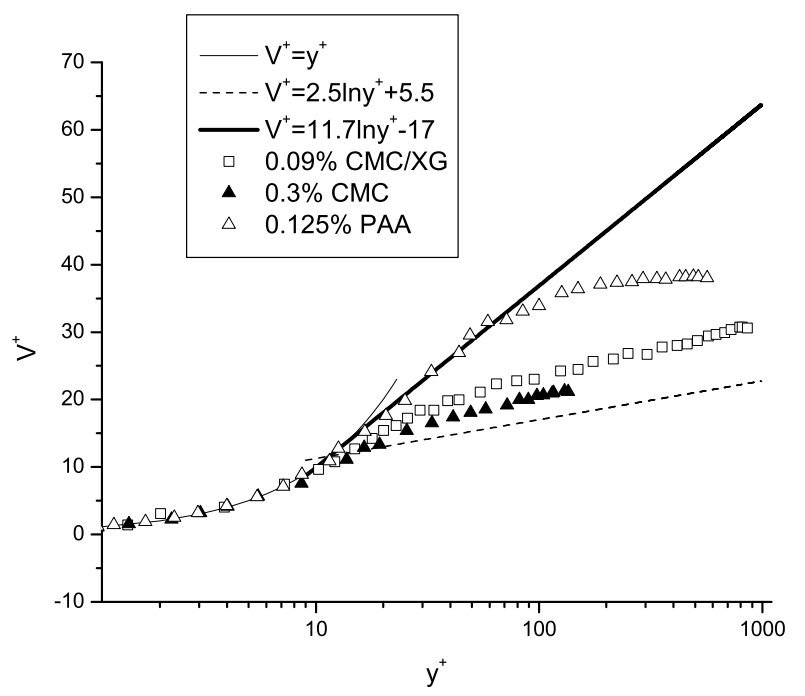

FIG. 1: Typical velocity profiles taken from 4]. In dashed line we noted the von-Kármán law (2), while the MDR (3) is shown as the continuous black line. In all cases the mean velocity follows the same viscous behavior for $y^{+}<10$. After that the scenario is different for flexible and rodlike polymers. The typical behavior for the former is presented by the open triangles, which follow the MDR up to a crossover point that depends on the concentration of the polymer and on the value of Re. The rodlike behavior is exemplified by the solid triangles and the open squares; the mean velocity profiles appear to interpolate smoothly between the two asymptotes as a function of the concentration of the rodlike polymer. ized coordinates. Denote the mean pressure (per unit density) gradient $p^{\prime} \equiv-\partial p / \partial x$ where $x, y$ and $z$ are the lengthwise, wall-normal and spanwise directions respectively. The length and width of the channel are usually taken much larger than the mid-channel height $L$, making the latter a natural re-scaling length for the introduction of dimensionless (similarity) variables, also known as "wall units" 3]. Thus the Reynolds number Re, the normalized distance from the wall $y^{+}$and the normalized mean velocity $V^{+}\left(y^{+}\right)$(which is in the $x$ direction with a dependence on $y$ only) are defined by

$$
\operatorname{Re} \equiv L \sqrt{p^{\prime} L} / \nu_{0}, y^{+} \equiv y \operatorname{Re} / L, V^{+} \equiv V / \sqrt{p^{\prime} L},
$$

where $\nu_{0}$ is the kinematic viscosity of the neat fluid. For Newtonian fluids the profile of the mean velocity $V^{+}\left(y^{+}\right)$ is universal, starting with the viscous sub-layer in which $V^{+}\left(y^{+}\right)=y^{+}$and then, at $y^{+}$somewhere between 6 and 12 , the profile crosses over to the universal von-Kármán log-law of the wall (cf. Fig. 11)

$$
V^{+}\left(y^{+}\right)=\kappa_{\mathrm{K}}^{-1} \ln y^{+}+B .
$$

Upon the addition of small concentrations of polymers, the drag is reduced and for the same value of $p^{\prime}$ one finds an increase in $V^{+}\left(y^{+}\right)$. This phenomenon exhibits both universal and non-universal aspects. The universal aspect is the Maximum Drag Reduction (MDR) asymptote, which is the largest attainable profile $V^{+}\left(y^{+}\right)$. This was determined experimentally by Virk who found 2]

$$
V^{+}\left(y^{+}\right)=\frac{1}{\kappa_{\mathrm{V}}} \ln \left(e \kappa_{\mathrm{v}} y^{+}\right) \quad \text { for } y^{+} \gtrsim 12 .
$$

While $\kappa_{\mathrm{K}}^{-1} \approx 2.5, \kappa_{\mathrm{v}}^{-1} \approx 12$, leading to a significantly larger mean velocity at the MDR as compared with vonKármán's log-law. The MDR appears independent of the nature of the polymer (for example whether it is flexible or rodlike), of the length of the polymer and of the concentration. On the other hand, the way that the system attains the MDR is not universal, and it depends on all of the above. In particular, it appears that flexible and rodlike polymers attain the MDR, as a function of the 
concentration, in qualitatively different ways (see for example [2]). The experimental information is quite scant, but available data indicate different scenarios for the two types of polymers. The data in Fig. Mindicate for large values of Re the mean velocity profile with flexible polymers [polyacrylamide (PAA)] follows the MDR until a point of cross over back to the "Newtonian plug", where it becomes parallel to von-Kármán's log-law. Increasing the concentration results in following the MDR further until a higher cross over point is attained back to the Newtonian plug [2]. On the other hand, for rodlike polymers [sodium carboxymethylcellulose (CMC) and sodium carboxymethylcellulose/xanthan gum blend (CMC/XG)] the data shown in Fig. 1 indicate a different scenario. Contrary to flexible polymers, here, as a function of the concentration, one finds mean velocity profiles that interpolate between the two asymptotes (2) and (3), reaching the MDR only for large concentrations. A difference in the behavior of flexible and rodlike polymers when plotting the drag as a function of Reynolds number was also reported by Virk and coworkers, see $[\underline{5}$.

The universal MDR was fully explained in recent work, and its parameters derived, by showing that it is an edge turbulent solution in a channel [6]. In other words, if one tried to reduce the drag further (or further increase the profile $V^{+}\left(y^{+}\right)$), one would lose the turbulent flow in favor of a laminar solution. This is the reason for the universality of the MDR and its insensitivity to the nature of the polymer. The aim of this paper is to derive quantitatively the non-universal scenarios of attaining the MDR by flexible and rodlike polymers. We will limit our attention to the case of high Re, and consider the profiles $V^{+}\left(y^{+}\right)$for different concentrations, with the aim of explaining the phenomenology displayed in Fig. 1 The paper ends with a definite prediction of different crossover behavior in the mean velocity profiles for flexible and rodlike polymers, in agreement with the indication of Fig. [1

In Sect. 2 we summarize the available theory of drag reduction by flexible and rodlike polymers, based on the balance equations for energy and momentum in the turbulent boundary layer. We derive in this section the equations satisfied by the $y$ dependence of the mean shear (in wall units), from which the velocity profiles are obtained by integration. Sect. 3 presents the results together with a summary and a discussion.

\section{THEORY OF DRAG REDUCTION}

\section{A. The polymeric stress tensor}

In the presence of a small concentration of polymers the Navier-Stokes equations for the fluid velocity $\boldsymbol{U}(\boldsymbol{r}, t)$ gain an additional stress tensor:

$$
\begin{aligned}
\frac{\partial \boldsymbol{U}}{\partial t}+\boldsymbol{U} \cdot \boldsymbol{\nabla} \boldsymbol{U} & =-\nabla p+\nu_{0} \nabla^{2} \boldsymbol{U}+\nabla \cdot \boldsymbol{\sigma}, \\
\nabla \cdot \boldsymbol{U} & =0 .
\end{aligned}
$$

The extra stress tensor $\boldsymbol{\sigma}$ is due to the interaction between the polymers and the fluid. Within the FENE-P model for flexible polymers we have [7]

$$
\sigma_{a b} \approx \nu_{p} \gamma_{p} \mathcal{R}_{a b},
$$

where $\nu_{p}$ is the polymeric contribution to the viscosity at zero shear, and $\gamma_{p}$ is the inverse relaxation time of the stretched polymer. In writing this expression one adopts the standard simplification of a single relaxation time. The conformation tensor $\mathcal{R}$ is obtained from the normalized end-to-end distance vector of the polymer $\hat{\boldsymbol{\rho}} \equiv \boldsymbol{\rho} / \rho_{\max }$, averaged over the conformation of the polymers,

$$
\mathcal{R} \equiv \overline{\hat{\rho} \hat{\rho}}
$$

For rodlike polymer $\hat{\boldsymbol{\rho}}$ becomes a unit vector, and there is no coil-stretching transition. Accordingly the stress tensor assumes a different form [8]:

$$
\sigma_{a b}=6 \nu_{p} \mathcal{R}_{a b} \mathcal{R}_{i j} S_{i j},
$$

where $S_{i j}$ is the strain experienced by the polymer $S_{i j} \equiv$ $\partial U_{i} / \partial r_{j}$. As explained above, the difference in form of the stress tensor is immaterial for the universal form of the MDR 2, 9], a phenomenon that was called "additive equivalence" by Virk. We will see that this difference translates however to a very different scenario for the attainment of the MDR.

\section{B. The balance equations}

The phenomenon of drag reduction can be understood on the basis of the balance equations of the mechanical momentum and turbulent energy [10]. These are derived on the basis of the Reynolds decomposition

$$
\begin{aligned}
U_{i}(\boldsymbol{r}, t) & =V(y) \delta_{i x}+u_{i}(\boldsymbol{r}, t) \\
S_{i j}(\boldsymbol{r}, t) & =S(y) \delta_{i x} \delta_{j y}+s_{i j}(\boldsymbol{r}, t), \quad S(y) \equiv \frac{d V(y)}{d y}(.9)
\end{aligned}
$$

Writing these equations we use the fact that in a turbulent channel flow $p^{\prime}$ is constant, and due to the symmetry all the other mean quantities depend on $y$ only. In addition to the mean shear $S(y)$, we need to to introduce the mean turbulent kinetic energy $K \equiv\left\langle u^{2}\right\rangle / 2$ and the Reynolds stress $W \equiv-\left\langle u_{x} u_{y}\right\rangle$. The momentum balance equation is obtained by averaging Eq. (44) and integrating with respect to $y$, ending up with the exact equation:

$$
\nu_{0} S+W+\left\langle\sigma_{x y}\right\rangle=p^{\prime}(L-y) .
$$

In wall-units Eq. (10) can be written in a more elegant form:

$$
S^{+}+W^{+}+\left\langle\sigma_{x y}^{+}\right\rangle=\left(1-y^{+} / \mathrm{Re}\right)
$$


where $S^{+} \equiv \nu_{0} S /\left(p^{\prime} L\right), W^{+} \equiv W /\left(p^{\prime} L\right)$, and $\sigma_{i j}^{+} \equiv$ $\sigma_{i j} /\left(p^{\prime} L\right)$. When Re is very large and for $y^{+}$not too large we neglect the second term on the RHS, approximating the RHS as unity.

The balance equation for the turbulent kinetic energy is calculated by taking the dot product of the fluctuation part of Eq. (4) with $\boldsymbol{u}$ :

$$
W S=\frac{\partial}{\partial y}\left\langle u_{y} u^{2}+u_{y} p-\sigma_{i y} u_{i}\right\rangle+\nu_{0}\left\langle s_{i j} s_{i j}\right\rangle+\left\langle\sigma_{i j} s_{i j}\right\rangle .
$$

Also this equation is exact. We simplify it by noting that the first term on the RHS involves the spatial flux of turbulent energy which is known to be negligible in the log-layer. The second term represents the dissipation which is modelled (in wall-units) as [10]:

$$
\left\langle s_{i j}^{+} s_{i j}^{+}\right\rangle \approx K^{+}\left(\frac{a}{y^{+}}\right)^{2}+b \frac{\left(K^{+}\right)^{3 / 2}}{y^{+}} .
$$

where $s_{i j}^{+} \equiv \nu_{0} s_{i j} /\left(p^{\prime} L\right)$ and $K^{+} \equiv K /\left(p^{\prime} L\right)$. Therefore, the energy balance equation takes on the form

$$
W^{+} S^{+}=K^{+}\left(\frac{a}{y^{+}}\right)^{2}+b \frac{\left(K^{+}\right)^{3 / 2}}{y^{+}}+\left\langle\sigma_{i j}^{+} s_{i j}^{+}\right\rangle
$$

Finally, we quote the experimental fact that in the loglayer $W^{+}$and $K^{+}$are proportional to each other:

$$
K^{+} c^{2}=W^{+} \text {. }
$$

Experimentally, it was found that $c \approx 0.5$ in the Newtonian case, and $c \approx 0.25$ in the MDR.

\section{Effect of the polymers}

In the Newtonian case, when $\boldsymbol{\sigma}=0$ and $c=0.5$, the three equations (11), (14) and (15) are sufficient for determining the three unknowns $S^{+}, K^{+}$and $W^{+}$. The best fit to Newtonian experiments and simulations are obtained using the values $a=3$ and $b=0.321$, and we are going to use these values throughout, also in the viscoelastic cases discussed below. In the presence of polymers, however, we have to consider the terms introduced by the polymers to these equations. The necessary theory was presented in [9, 10], with the final results relating these terms to the yy component of the mean conformation tensor $\boldsymbol{R} \equiv\langle\boldsymbol{\mathcal { R }}\rangle$ : .

$$
\begin{aligned}
\left\langle\sigma_{x y}\right\rangle & \approx \nu_{p} R_{y y} S, \\
\left\langle\sigma_{i j} s_{i j}\right\rangle & \approx \nu_{p} R_{y y} \frac{K}{y^{2}} .
\end{aligned}
$$

Using these theoretical results the momentum and energy equations, in the limit of large Re, are reduced to

$$
S^{+}+W^{+}+\tilde{\nu} R_{y y} S^{+}=1
$$

and

$$
W^{+} S^{+}=K^{+}\left(\frac{a}{y^{+}}\right)^{2}+b \frac{\left(K^{+}\right)^{3 / 2}}{y^{+}}+\tilde{\nu} R_{y y} K^{+}\left(\frac{a}{y^{+}}\right)^{2} .
$$

Here $\tilde{\nu}=\nu_{p} / \nu_{0}$. To proceed we need to relate $R_{y y}$ to the other variables. The necessary theory is presented in 7, 9, 10, 11] leading to an important difference between the flexible and the rodlike polymers:

$$
\begin{array}{ll}
R_{y y} \approx \frac{\sqrt{K}}{S y} & \text { (flexible) } \\
R_{y y} \approx \frac{K}{S^{2} y^{2}} & \text { (rodlike) }
\end{array}
$$

Substituting into Eqs. (17) and (18) we have

$$
\nu_{\mathrm{eff}} S^{+}+W^{+}=1
$$

and

$$
W^{+} S^{+}=K^{+} \nu_{\mathrm{eff}}\left(\frac{a}{y^{+}}\right)^{2}+b \frac{\left(K^{+}\right)^{3 / 2}}{y^{+}}
$$

with the "effective viscosity"

$$
\begin{array}{ll}
\nu_{\mathrm{eff}}=1+\tilde{\nu} \frac{\sqrt{K^{+}}}{S^{+} y^{+}} & \text {(flexible) } \\
\nu_{\mathrm{eff}}=1+\tilde{\nu} \frac{K^{+}}{\left(S^{+} y^{+}\right)^{2}} & \text { (rodlike) } .
\end{array}
$$

In the next section we will show that although the effective viscosities take on different forms for the flexible and rodlike cases, in fact they both depend linearly on $y^{+}$ whenever we have a $\log$ layer with $S^{+} \propto 1 / y^{+}$. The reason is that $K^{+}$turns out to be proportional to $\left(y^{+}\right)^{2}$ and to $y^{+}$for the flexible and the rodlike cases respectively. Accordingly, we will write in both cases

$$
\nu_{\mathrm{eff}}=1+\alpha\left[y^{+}-\Delta(\alpha)\right],
$$

with $\Delta(\alpha)$ being the width of the viscous sub-layer, and its dependence on the slope of the effective viscosity $\alpha$ needs to be determined. It is natural to present $\Delta(\alpha)$ in terms of a dimensionless scaling function $f(x)$,

$$
\Delta(\alpha)=\delta^{+} f\left(\alpha \delta^{+}\right)
$$

where $\delta^{+} \approx 6$ is the width of the Newtonian viscous boundary layer. In the Newtonian limit $\alpha \rightarrow 0, \nu_{\text {eff }} \rightarrow 1$ and $\Delta \rightarrow \delta^{+}$, hence we have $f(0)=1$. In [6] it was shown that the balance equations (21) and (22) (with the prescribed form of the effective viscosity profile) have an non-trivial symmetry that leaves them invariant under rescaling of the wall units. This symmetry dictates the function $\Delta(\alpha)$ in the form

$$
\Delta(\alpha)=\frac{\delta^{+}}{1-\alpha \delta^{+}} .
$$




\section{Closing the equations}

To complete the model, we have to specify the value of $c$ in Eq. (15). This parameter becomes naturally a function of $\alpha$. We can find its $\alpha$-dependence by identifying the width of the viscous sub-layer $\Delta$ with $a / c(\alpha)$. This stems from the fact that the balance equations cannot support a turbulent solution for $y^{+}<a / c(\alpha)$. This means that

$$
a / c(\alpha)=\Delta(\alpha)
$$

Combining then Eqs. (25), (27) and (28), and putting $\delta^{+}=6$, we can solve and find

$$
\begin{aligned}
c\left(\nu_{\mathrm{eff}}\right) & =\frac{a}{6}+\frac{a\left(2-\nu_{\mathrm{eff}}\right)}{2 y^{+}} \\
& +\frac{a}{12}\left[\sqrt{1-\frac{12 \nu_{\mathrm{eff}}}{y^{+}}+\frac{36\left(2-\nu_{\mathrm{eff}}\right)^{2}}{\left(y^{+}\right)^{2}}}-1\right]
\end{aligned}
$$

To summarize note that Eqs. (23) and (24) can be written as

$$
K^{+}=A^{2}\left(S^{+} y^{+}\right)^{2}
$$

with

$$
\begin{array}{ll}
A^{2}=\left(\frac{\nu_{\mathrm{eff}}-1}{\tilde{\nu}}\right)^{2} & (\text { flexible }) \\
A^{2}=\frac{\nu_{\mathrm{eff}}-1}{\tilde{\nu}} & (\text { rodlike })
\end{array}
$$

Using Eqs. (15) and (30), we can rewrite Eqs. (21) and (22) as two equations for the two variables $\nu_{\text {eff }}$ and $S^{+}$:

$$
\nu_{\mathrm{eff}} S^{+}+c^{2} A^{2}\left(S^{+} y^{+}\right)^{2}=1,
$$

and

$$
c^{2} S^{+}=\nu_{\mathrm{eff}}\left(\frac{a}{y^{+}}\right)^{2}+b A S^{+}
$$

Equation (34) implies

$$
S^{+}=\frac{\nu_{\mathrm{eff}}}{\left(y^{+}\right)^{2}} \frac{a^{2}}{\left(c^{2}-b A\right)}
$$

Substituting Eq. (35) into Eq. (33) gives an equation for $\nu_{\text {eff }}$ :

$$
\nu_{\mathrm{eff}}^{2}\left(\frac{a}{y^{+}}\right)^{2}\left(c^{2}-b A\right)+c^{2} A^{2} \nu_{\mathrm{eff}}^{2}\left(\frac{a^{2}}{y^{+}}\right)^{2}=\left(c^{2}-b A\right)^{2}
$$

Finally, we can solve Eq. (36) to get $\nu_{\text {eff }}\left(y^{+}\right)$for different values of $\tilde{\nu}$. Then we can obtain $S^{+}$and $K^{+}$using Eqs. (35) and (30) respectively. Integrating $S^{+}$over $y^{+}$, we get also $V^{+}\left(y^{+}\right)$.

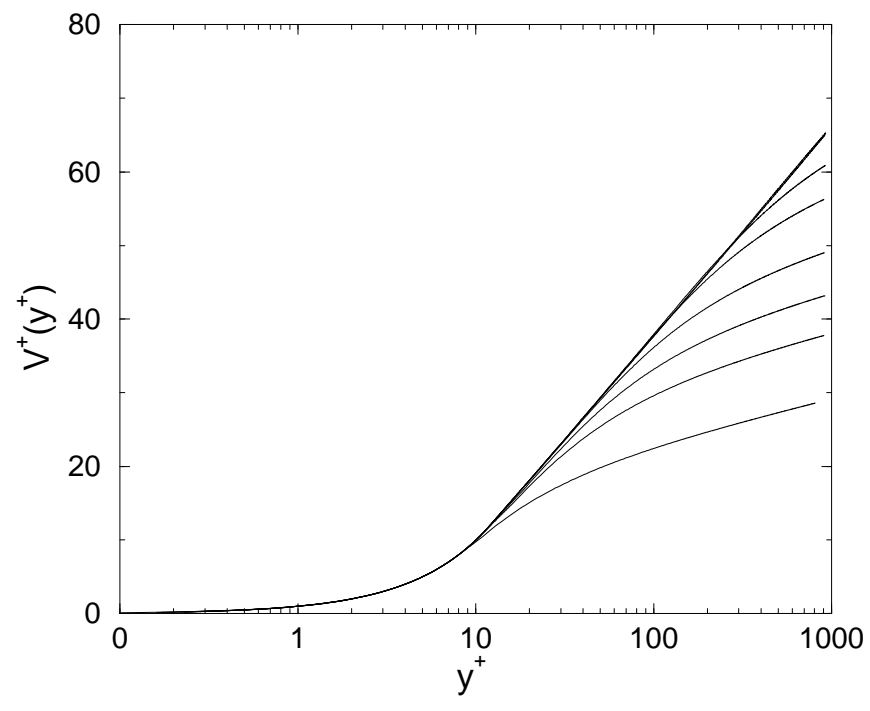

FIG. 2: The mean velocity profiles for flexible polymer additives with $\tilde{\nu}=1,5,10,20,50,100$ and 500 from below to above. Note that the profile follows the MDR until it crosses over back to the Newtonian plug.

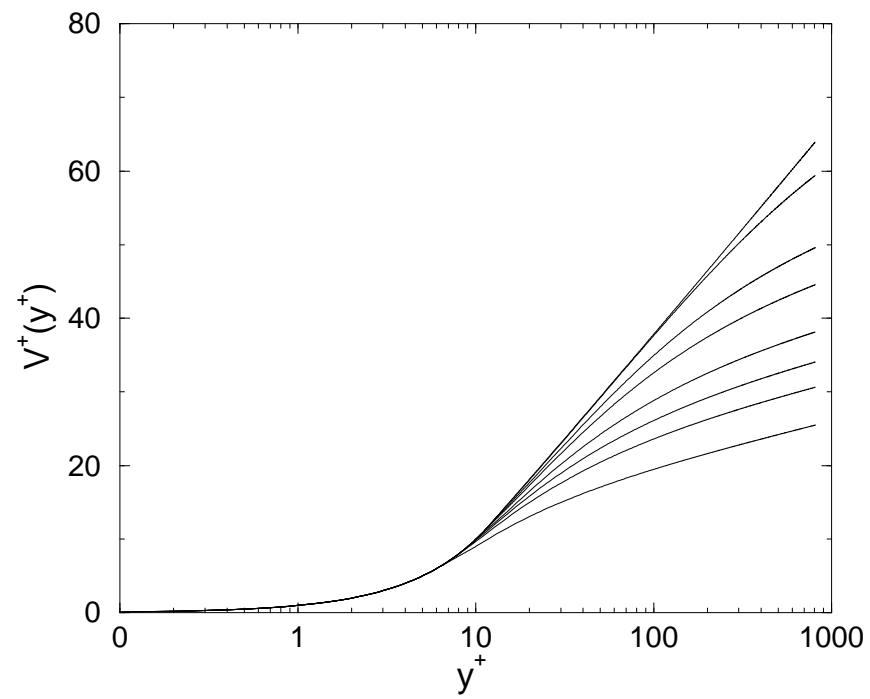

FIG. 3: The mean velocity profile for rodlike polymer additives with $\tilde{\nu}=1,5,10,20,50,100,500,1000,5000$ and 10000 from below to above. Note the typical behavior expected for rodlike polyemrs, i.e. that the profile diverges from the von Kármán log-law, reaching the MDR only asymptotically.

\section{RESULTS}

The results of the numerical solutions of the equations are shown in Figs. 2 [3 and 4

In Figs. 2 and 3 we present the mean velocity profile as a function of the distance from the wall, for flexible and rodlike polymers respectively. The main result of this paper is seen in the difference between these profiles as a function of the polymer concentration. While the flexible polymer case exhibits the feature [2, 5] that the velocity 


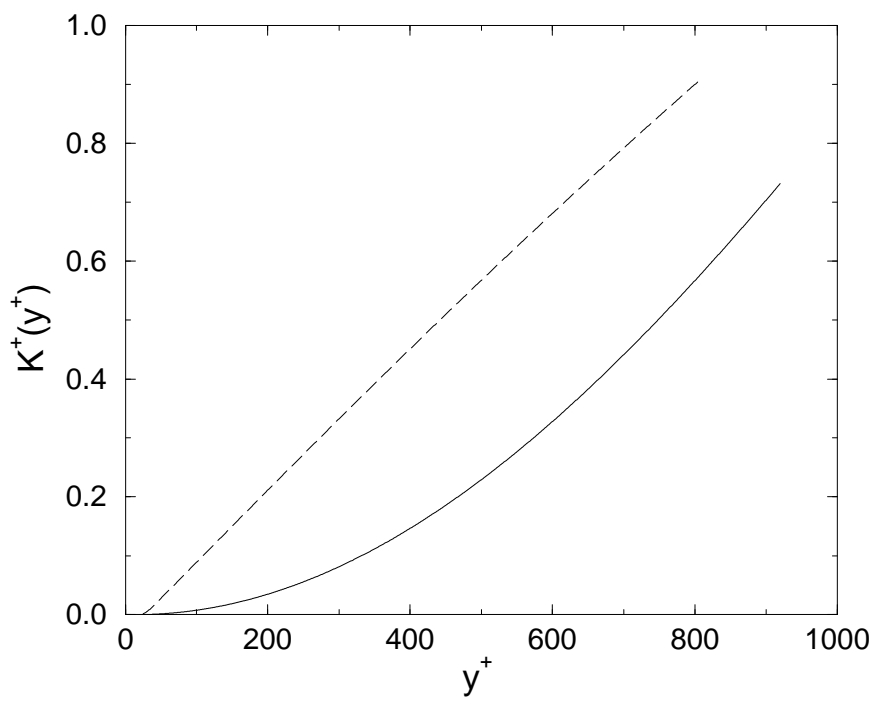

FIG. 4: The turbulent kinetic energy profile $K^{+}\left(y^{+}\right)$for the flexible (solid line) and rodlike (dashed line) cases respectively. We note the quadratic and linear dependence respectively, as anticipated in the text.

profile adheres to the MDR until a crossover to the Newtonian plug is realized, the rodlike case presents a "diverging" of profiles which only asymptotically reach the MDR. We also notice that the flexible polymer matches the MDR faithfully for relatively low values of $\tilde{\nu}$, whereas the rodlike case attains the MDR only for much higher values of $\tilde{\nu}$. This result is in agreement with the experimental finding in [12, 13] that the flexible polymer is a better drag reducer than the rodlike analogue.

We should note that the higher efficacy of flexible polymers cannot be easily related to their elongational viscosity as measured in laminar flows. In some studies [12, 13, 14] it was proposed that there is a correlation between the elongational viscosity measured in laminar flows and the drag reduction measured in turbulent flows. We find here that flexible polymers do better in turbulent flows due to their contribution to the effective shear viscosity, and their improved capability in drag reduction stems simply from their ability to stretch, something that rodlike polymers cannot do.

Finally we recall that the derivation of our equations relied on the fact that the turbulent kinetic energy $K^{+}$ depends linearly and quadratically on the distance from the wall for flexible and rodlike polymers respectively. It is important to check that the resulting equations confirm this expectation self-consistently. Indeed, in Fig. [4 we present the solution for this quantity in the two cases, and find that the expectation is fully realized.

\section{Acknowledgments}

This work has been supported in part by the European Commission under a TMR grant and by the Research Grants Council of Hong Kong (CUHK 400304).
[1] A. Gyr and H. W. Bewersdorff Drag Reduction of Turbulent Flows by Additives (Kluwe, London, 1995).

[2] P. S. Virk, AlChE Journal 21, 625 (1975).

[3] S.B. Pope, Turbulent Flows (Cambridge, 2000).

[4] M. P. Escudier, F. Presti and S. Smith, J. Non-Newtonian Fluid Mech. 81, 197 (1999).

[5] P.S. Virk, D.C. Sherma and D.L. Wagger, AIChE Journal, 43, 3257 (1997).

[6] R. Benzi, E. deAngelis, V. S. L'vov and I. Procaccia, Phys. Rev. Lett., 95, 194502 (2005).

[7] R. Benzi, E. de Angelis, V. S. L'vov, I. Procaccia and V. Tiberkevich, J. Fluid Mech. in press.

[8] M. Doi and S. F. Edwards The Theory of Polymer Dynamics (Oxford, 1988).
[9] R. Benzi, E. S.C. Ching, T. S. Lo, V. S. L'vov, and I. Procaccia, Phys. Rev. E., 72, 016305(2005).

[10] V. S. L'vov, A. Pomyalov, I. Procaccia and V. Tiberkervich, Phys. Rev. Lett., 94, 174502 (2005)

[11] V. S. L'vov, A. Pomyalov, I. Procaccia and V. Tiberkevich, Phys. Rev. E., 71, 016305(2005).

[12] C. Wagner, Y. Amarouchène, P. Doyle and D. Bonn, Europhys. Lett. 64, 823 (2003).

[13] D. Bonn, Y. Amarouchǹe, C. Wagner, S. Douady and O. Cadot, J. Phys.: Condens. Matter, 17, S1195 (2005).

[14] J. M. J. den Toonder, F. T. M. Nieuwstadt and G. D. C. KuiKen , Appl Sci Res, 54, 95 (1995). 